\title{
Effects of parenteral glutamine supplementation on modulating the immune response in rats undergoing a total gastrectomy
}

\author{
Ming-Tsan Lin ${ }^{1}$, Szu-Yuan $\mathrm{Chou}^{2}$, Shung-Sheng Tsou ${ }^{3}$, Ming-Yang Wang ${ }^{1}$, Ming-Hsun $\mathrm{Wu}^{1}$ \\ and Sung-Ling $\mathrm{Yeh}^{4} *$ \\ ${ }^{1}$ Department of Surgery, College of Medicine, National Taiwan University Hospital and National Taiwan University, Taipei, \\ Taiwan, ROC \\ ${ }^{2}$ Department of Obstetrics and Gynecology, Taipei Medical University-Associated Wan Fang Hospital and Shuang Ho Hospital, \\ Taipei, Taiwan, ROC \\ ${ }^{3}$ Department of Surgery, Tung's Taichung MetroHarbor Hospital, Taichung, Taiwan, ROC \\ ${ }^{4}$ School of Nutrition and Health Sciences, Taipei Medical University, 250 Wu-Hsing Street, Taipei 110, Taiwan, ROC
}

(Received 4 June 2008 - Revised 12 December 2008 - Accepted 15 December 2008 - First published online 10 February 2009)

The present study investigated the effect of parenteral glutamine (Gln) supplementation on cellular adhesion molecule expression and release of chemokines responsible for inflammatory cell recruitment in rats undergoing a total gastrectomy. Normal rats with internal jugular catheters were assigned to one control group and two experimental groups and received total parenteral nutrition (TPN). A total gastrectomy was performed in the experimental groups, whereas the control group received a sham operation (Sham). The TPN solutions were isonitrogenous and identical in nutrient composition except that the Sham group and one of the experimental group received conventional (Conv) TPN solution, whereas the other experimental group received $25 \%$ of the amino acid nitrogen as Gln. Half of the rats in each group were killed 1 or $3 \mathrm{~d}$ after surgery or the Sham to examine their immune response. The results showed that the surgery produced higher polymorphonuclear leucocyte CD11b/CD18 expressions, and Gln supplementation lowered CD11b/CD18 expressions compared with the Conv group post-operatively. The levels of monocyte chemotactic protein-1 and macrophage inflammatory protein-2 in peritoneal lavage fluid were higher in the Gln group than those in the Conv group $1 \mathrm{~d}$ post-operatively; these chemotactic proteins had returned to the levels comparable with those in the Sham group on post-operative day 3 . These results suggest that Gln supplementation attenuated polymorphonuclear leucocyte integrin expression. In addition, Gln-enriched parenteral nutrition induced an earlier more intensive and rapid immune response to injury than the Conv parenteral nutrition after a total gastrectomy.

Total gastrectomy: Glutamine: CD11b/CD18: Monocyte chemotactic protein: Macrophage inflammatory protein

Gastric cancer is among the top ten causes of cancer-related deaths for both men and women in many countries, especially in Asia. A total gastrectomy is a major abdominal surgical procedure, which is frequently undertaken for malignant gastric diseases and usually causes much stress to patients. For most gastrectomised patients with gastric diseases, preoperative malnutrition often occurs and adequate post-operative oral intake is achieved late ${ }^{(1,2)}$. Enteral and parenteral nutritional support is necessary for such patients. Although studies have shown that early enteral feeding seems to be well tolerated by patients ${ }^{(3)}$, most surgeons use the parenteral route to administer nutrients before and after a gastrectomy due to preoperative obstruction or post-operative ileus. The optimal parenteral formulation for patients undergoing a total gastrectomy is still being investigated.

Glutamine (Gln) is an abundant free amino acid in the circulation. It has traditionally been considered a non-essential amino acid, but is now known to be conditionally essential following critical illness and injury ${ }^{(4,5)}$. Previous studies demonstrated numerous benefits of Gln administration in the experimental models of serious metabolic stress, including attenuated proinflammatory cytokine expression, improved gut barrier function and enhanced immune cell function ${ }^{(6-10)}$. A meta-analysis of several studies reviewed by Novak et al. ${ }^{(11)}$ concluded that in surgical patients, Gln supplementation demonstrated a trend towards a benefit in reducing infectious complications and the length of the hospital stay. The mortality benefit was even more striking in the trials that used Gln in the parenteral route ${ }^{(11)}$. However, the efficacy of parenteral Gln administration on major surgery as total gastrectomy has not been evaluated.

Major surgery has been shown to result in an inflammatory reaction. Inflammatory mediators including adhesion molecules and chemokines are expressed to recruit inflammatory

\footnotetext{
Abbreviations: BW, body weight; CINC, cytokine-induced neutrophil chemoattractant; Conv, conventional; Gln, glutamine; IFN, interferon; MCP, monocyte chemotactic protein; MIP, macrophage inflammatory protein; PMN, polymorphonuclear neutrophil; Sham, control group received a sham operation; TPN, total parenteral nutrition.
}

* Corresponding author: Sung-Ling Yeh, fax +886 2 27373112, email sangling@tmu.edu.tw 
cells to the site of injury ${ }^{(12-15)}$. Besides, surgery is known to impair immune response ${ }^{(16)}$, and the Th2 cytokine is associated with operation-induced immunosuppression ${ }^{(17)}$. Previous studies performed by our laboratory showed that Gln supplementation reduced adhesion molecule expression and neutrophil infiltration into tissues ${ }^{(10)}$; besides, a more balanced Th1/Th2 response was found during sepsis when Gln was administered $^{(18)}$. However, the effect of Gln on leucocyte adhesion molecule expression and inflammatory cell recruitment after major surgery has not clearly been elucidated. We hypothesised that parenterally infused Gln would reduce leucocyte adhesion molecule expression and release of chemokines responsible for inflammatory cell recruitment after surgery. $\beta 2$ Integrins (CD18) are surface proteins expressed on leucocytes and are important in the adhesion of leucocytes to activated endothelium ${ }^{(19)}$. Integrins are reported to be good markers of leucocyte activation in the inflammatory mechanism $^{(20)}$. Cytokine-induced neutrophil chemoattractant (CINC)-1 and macrophage inflammatory protein (MIP)-2 play important roles in mediating neutrophil recruitment to the site of injury ${ }^{(13,14)}$. Monocyte chemotactic protein (MCP)-1 is a chemotactic and activating factor for mononuclear phagocytes. MCP-1 is also involved in recruiting peripheral blood leucocytes to the peritoneal cavity ${ }^{(15)}$. In order to determine the possible roles of Gln in modulating inflammatory mediators in a surgical condition, the levels of these adhesion molecules and chemokines were measured. Also, the regulatory role of Gln on the Th1/Th2 response after major surgery was evaluated in the present study.

\section{Materials and methods}

\section{Animals}

Male 7-week-old Wistar rats weighing 180-210g at the beginning of the experiment were used. All rats were housed in temperature- and humidity-controlled rooms and were allowed free access to standard rat chow for $7 \mathrm{~d}$ before the experiment. The present study was approved by the National Taiwan University Hospital Animal Care Committee. The care of the animals was in full compliance with the Guide for the Care and Use of Laboratory Animals (National Research Council, 1985), as reviewed by the Animal Care and Use Committee of the National Taiwan University Hospital.

\section{Study protocol and operative procedures}

Rats were randomly assigned to one control group and two experimental groups, with each group containing twenty rats. The average weights among the groups were adjusted to be as similar as possible. After overnight starvation, all rats (including the control and experimental groups) were anesthetised with intraperitoneal pentobarbital sodium $(50 \mathrm{mg} / \mathrm{kg})$, and the right internal jugular vein was cannulated with a Silastic catheter (Dow Corning, Midland, MI, USA) under sterile conditions. At the same time, a total gastrectomy was performed in the experimental groups, while the control group received a sham operation (Sham) group. Sham was performed by a laparotomy with a $4 \mathrm{~cm}$ midline incision. The stomach was pulled out and placed back, and then the cavity was closed in two layers. In experimental groups, a total gastrectomy was carried out through a $4 \mathrm{~cm}$ midline incision, as described by Ohta et al. ${ }^{(21)}$. Briefly, the stomachs of the rats were removed after ligation of most of the blood vessels around the stomach, which supplied blood, and an end-to-side anastomosis was carried out between the cut edge of the oesophagus and the upper jejunum, $5 \mathrm{~cm}$ distal from the ligament of Treitz. Finally, the cavity was sutured closed in two layers. Half of the rats in each group ( $n$ 10) were killed 1 or $3 \mathrm{~d}$ (days 2 and 4 of TPN) after the operation. TPN was begun immediately after the operation and was maintained for 2 or $4 \mathrm{~d}$ in the different groups according to the killing schedule of the rats. All basal TPN solutions were isonitrogenous $(6.82 \mathrm{mg} / \mathrm{ml})$ and identical in nutrient composition except for the difference in the amino acid content. The control group and one of the experimental groups were infused with Conv TPN group. The other experimental group was supplemented with Gln group (Dipeptiven; Fresenius Kabi, Bad Homburg, Germany), which provided $25 \%$ of the total amino acid nitrogen in the TPN solution. The amount of Gln administered was $2.97 \mathrm{~g} / \mathrm{kg}$ body weight (BW)/d. According to the BW of individual rat, it would be $0.53-0.62 \mathrm{~g} \mathrm{Gln} / \mathrm{rat}$. The TPN provided $1129.68 \mathrm{~kJ} / \mathrm{kg} \mathrm{BW} / \mathrm{d}$ $(270 \mathrm{kcal} / \mathrm{kg} \mathrm{BW} / \mathrm{d})$, and the energy $(\mathrm{kJ}): \mathrm{N}(\mathrm{g})$ ratio was 493.71:1. The energy density was $3.89 \mathrm{~kJ} / \mathrm{ml}(0.93 \mathrm{kcal} / \mathrm{ml})$. The energy distribution of the TPN solutions in the groups was $58 \%$ from glucose, $22 \%$ from protein and $20 \%$ from fat (Lipovenous 10\%; Fresenius AG; Table 1). The TPN solution was refilled daily and infused for $24 \mathrm{~h}$ at room temperature. Two millilitres per hour were administered on the first day, and then the rats received (200.832-238.488 kJ/d (48$57 \mathrm{kcal} / \mathrm{d})$ ) according to individual $\mathrm{BW}$. The infusion rate was maintained with a Terufusion pump (model STC-503; Terumo, Tokyo, Japan). The TPN solution without fat was prepared every other day in a laminar flow hood, and the fat emulsion was added daily just before use.

Table 1. Composition of the total parenteral nutrition solution $(\mathrm{ml} / \mathrm{l})$

\begin{tabular}{lcc}
\hline & Gln supplemented & Conventional \\
\hline Glucose $50 \%$ & 350 & 340 \\
Intralipid $20 \%$ & 100 & 100 \\
Moriamin-SN $10 \%{ }^{*}$ & 348 & 556 \\
Dipeptiven $20 \%$ & 82 & - \\
Infuvita† & 8 & 8 \\
$\mathrm{NaCl} \mathrm{3 \%}$ & 35 & 35 \\
$\mathrm{KCl} 7 \%$ & 10 & 10 \\
$\mathrm{~K}_{3} \mathrm{PO}_{4} 8.7 \%$ & 10 & 10 \\
$\mathrm{Ca}_{\text {gluconate }}$ & 10 & 10 \\
$\mathrm{MgSO}_{4}$ & 4 & 4 \\
Zn SO & 2 & 2 \\
Choline chloride (g) & 1 & 1 \\
\hline
\end{tabular}

Gln, glutamine.

${ }^{*}$ From Chinese Pharmaceuticals, Taipei, Taiwan. Contents (mg/dl): Leu 1250, Ile 560, Lys acetate 1240, Met 350, Phe 935, Thr 650, Trp 130, Val 450, Ala 620, Arg 790, Asp 380, Cys 100, Glu 650, His 600, Pro 330, Ser 220, Tyr 35 and aminoacetic acid (Gly) 1570.

†From Yu-Liang Pharmaceuticals, Taoyuan, Taiwan. Contents per ml: ascorbic acid, $20 \mathrm{mg}$; vitamin A, $660 \mathrm{IU}$ (198 $\mu \mathrm{g}$ retinol); ergocalciferol, $40 \mathrm{IU}(1 \mu \mathrm{g})$; thiamine $\mathrm{HCl}, 0.6 \mathrm{mg}$; riboflavin, $0.72 \mathrm{mg}$; niacinamide, $8 \mathrm{mg}$; pyridoxine $\mathrm{HCl}, 0.8 \mathrm{mg}$; D-panthenol, $3 \mathrm{mg}$; DL- $\alpha$-tocopheryl acetate, $2 \mathrm{mg}$; biotin, $12 \mu \mathrm{g}$; folic acid, $80 \mu \mathrm{g}$ cyanocobalamin, $1 \mu \mathrm{g}$. 


\section{Measurements and analytical procedures}

Rats in the respective groups were killed 1 or $3 \mathrm{~d}$ after surgery. Animals were anesthetised with intraperitoneal pentobarbital sodium $(50 \mathrm{mg} / \mathrm{kg} \mathrm{BW})$. A middle abdominal incision was made, and $10 \mathrm{ml}$ of PBS was intraperitoneally injected to elute the peritoneal cells. After harvesting the peritoneal lavage fluid, rats were killed by drawing arterial blood from the aorta. Blood samples were collected in tubes containing heparin. Fresh whole blood was used for the flow cytometric analysis.

\section{Analysis of CD11a/CD18 distributions in lymphocytes and polymorphonuclear neutrophil expressions of CD11b/CD18}

Hundred microlitres of fresh blood were incubated with $10 \mu \mathrm{l}$ of fluorescein isothiocyanate-conjugated monoclonal mouse anti-rat CD11a and phycoerythrin-conjugated mouse anti-rat CD18 (Serotec, Oxford, UK) antibodies for $15 \mathrm{~min}$ at $4^{\circ} \mathrm{C}$. Afterwards, red blood cells were lysed with lysing buffer (Serotec). The proportions of CD11a/CD18 expressed on lymphocytes were analysed by flow cytometry (Coulter, Miami, FL, USA). Fluorescence data were collected, and the results are presented as a percentage of CD11a-presenting cells in $1 \times 10^{5}$ lymphocytes. To determine CD11b/CD18 expressions on polymorphonuclear neutrophils (PMN), fluorescein isothiocyanate-conjugated mouse monoclonal anti-rat $\mathrm{CD} 11 \mathrm{~b}$ and phycoerythrin-conjugated mouse anti-rat CD18 (Serotec) antibodies were added to $100 \mu \mathrm{l}$ of the PMN suspension. Fluorescence data were collected on $1 \times 10^{5}$ viable cells and also analysed by flow cytometry (Coulter). The results are presented as a percentage of CD11b-presenting cells in $1 \times 10^{5}$ PMN. Lymphocytes and PMN were gated on the basis of their forward- and side-scatter profiles and analysed for the expressions of CD11a/CD18 and CD11b/CD18, respectively.

\section{Analysis of lymphocyte IL-4 and interferon- $\gamma$ expressions}

Populations of lymphocyte IL-4 and interferon (IFN)- $\gamma$ expressions in fresh blood were analysed by flow cytometry (Coulter). After killing the rats, $50 \mu \mathrm{l}$ of fresh blood was immediately incubated with Leucoperm (Serotec) to fix and penetrate the leucocytes, and then $10 \mu \mathrm{l}$ of fluorescein-conjugated mouse monoclonal anti-rat IFN- $\gamma$ (Serotec) and $5 \mu \mathrm{l}$ phycoerythrin-conjugated mouse monoclonal anti-rat IL-4 (Serotec) antibodies were incubated together for $30 \mathrm{~min}$. Lymphocytes capable of IL-4 and IFN- $\gamma$ expressions were assessed using dual intracellular cytokine staining and flow cytometry (Coulter). Lymphocytes were gated on the basis of their forward- and side-scatter profiles. The results are presented as a percentage of cytokine-producing cells in $1 \times 10^{5}$ lymphocytes.

Monocyte chemotactic protein-1, macrophage inflammatory protein-2 and cytokine-induced neutrophil chemoattractant-1 levels in peritoneal lavage fluid

MCP-1, MIP-2 and CINC-1 concentrations were measured by a quantitative sandwich enzyme immunoassay kit (BioSource, Camarillo, CA, USA; Amersham Pharmacia Biotech, Amersham, Buckinghamshire, UK). A monoclonal antibody specific for rat MCP-1, MIP-2 and CINC-1 was precoated onto a microplate. All procedures followed the manufacturer's instruction. The detection limits were $8,1.5$ and $1.3 \mathrm{pg} / \mathrm{ml}$ for MCP-1, MIP-2 and CINC-1, respectively.

\section{Statistical analysis}

Data are expressed as the means and standard deviations. Differences among groups with different diets were analysed by one-way ANOVA using Duncan's test. The paired $t$ test was used to compare the difference between 1 and $3 \mathrm{~d}$ postoperatively in the same group. $P<0.05$ was considered statistically significant.

\section{Results}

There were no differences in the initial BW and BW after TPN administration for $3 \mathrm{~d}$ among the groups (data not shown).

\section{Lymphocyte CD11a/CD18 and polymorphonuclear neutrophils CD11b/CD18 expressions}

There were no differences in lymphocyte CD11a/CD18 expressions among the three groups on any post-operative days. However, Conv group presented lower CD11a/CD18 expression on post-operative day 3 than day 1 . The Sham group had the lowest CD11b/CD18 expressions among the three groups on post-operative day 1 . The Conv group had higher $\mathrm{CD} 1 \mathrm{~b} / \mathrm{CD} 18$ expressions than the other two groups on post-operative days 1 and 3. The CD11b/CD18 expressions in the Conv and Gln groups on post-operative day 3 were lower than those on post-operative day 1 (Table 2).

\section{Lymphocyte interferon- $\gamma$ and IL-4 distributions}

The Gln group had lower IFN- $\gamma$ and IL-4 distributions than the other two groups on post-operative day 3 . The percentage of IL-4 in the Gln group was lower on day 3 than that on day 1 (Table 3).

Table 2. Lymphocyte CD11a/CD18 and polymorphonuclear neutrophils CD11b/CD18 expressions among the sham and experimental groups 1 and $3 \mathrm{~d}$ after the surgery

(Mean values and standard deviations for ten rats in each group)

\begin{tabular}{|c|c|c|c|c|}
\hline & \multicolumn{2}{|c|}{ CD11a/CD18 (\%) } & \multicolumn{2}{|c|}{ CD11b/CD18 (\%) } \\
\hline & Mean & SD & Mean & SD \\
\hline \multicolumn{5}{|l|}{ Post-1 } \\
\hline Sham & $11 \cdot 78$ & 1.42 & 1.92 & $1 \cdot 18$ \\
\hline Conv & 13.04 & 1.55 & $16 \cdot 9^{*}$ & 1.45 \\
\hline Gln & $9 \cdot 12$ & 1.35 & $11 \cdot 16$ & $1 \cdot 16$ \\
\hline \multicolumn{5}{|l|}{ Post-3 } \\
\hline Sham & $7 \cdot 62$ & 1.73 & $2 \cdot 80$ & 1.68 \\
\hline Conv & $7.68 \dagger$ & 1.52 & $10 \cdot 75^{\star} \dagger$ & 1.14 \\
\hline Gln & $8 \cdot 61$ & 1.49 & $3.43 \dagger$ & 1.15 \\
\hline
\end{tabular}

Post-1, experimental group $1 \mathrm{~d}$ after surgery; Sham, sham operation; Conv, conventional; Gln, glutamine; Post-3, experimental group $3 \mathrm{~d}$ after surgery.

* Mean values were significantly different from the other groups on the same postoperative day $(P<0.01)$.

† Mean values were significantly different from the same group on post-operative day $1(P<0.05)$. 
Table 3. Distributions of lymphocyte interferon (IFN)- $\gamma$ and IL-4 expressions among the sham and experimental groups 1 and $3 \mathrm{~d}$ after surgery

(Mean values and standard deviations for ten rats in each group)

\begin{tabular}{lccccc}
\hline & \multicolumn{2}{c}{ IFN- $\gamma(\%)$} & & \multicolumn{2}{c}{$\mathrm{IL}-4$ (\%) } \\
\cline { 2 - 3 } \cline { 6 - 6 } & Mean & SD & & Mean & SD \\
\hline Post-1 & & & & \\
Sham & 8.48 & 1.26 & & 9.83 & 2.18 \\
Conv & 7.78 & 1.12 & & 10.04 & 1.88 \\
Gln & 5.48 & 1.15 & & 10.62 & 1.54 \\
Post-3 & & & & \\
Sham & 9.07 & 1.63 & & 6.96 & 2.17 \\
Conv & 10.71 & 1.15 & & 7.03 & 1.51 \\
Gln & $7.20^{*}$ & 1.13 & & $2.95^{*} \dagger$ & 1.54 \\
\hline
\end{tabular}

Post-1, experimental group $1 \mathrm{~d}$ after surgery; Sham, sham operation; Conv, conventional; Gln, glutamine; Post-3, experimental group $3 \mathrm{~d}$ after surgery.

* Mean values were significantly different from the Sham and Conv groups on the same post-operative day $(P<0.05)$.

$\dagger$ Mean values were significantly different from the Gln group on post-operative day $1(P=0.006)$.

Monocyte chemotactic protein-1, macrophage inflammatory protein-2 and cytokine-induced neutrophil chemoattractant-1 levels in peritoneal lavage fluid

MCP-1 concentrations in the Gln group were higher than those of the Sham and Conv groups; the MIP-2 levels were also higher in the Gln group than that of the Conv group, but did not differ from that of the Sham group on post-operative day 1. The CINC-1 and MIP-2 levels in peritoneal lavage fluid were significantly higher in the Conv group than those in the Sham and Gln groups on post-operative day 3. The Conv group had higher MIP-2 on post-operative day 3 than that on day 1 . The concentration of MCP-1 was significantly lower in the Gln group on day 3 than that on day 1 postoperatively (Table 4).

Table 4. Concentrations of cytokine-induced neutrophil chemoattractant (CINC)-1, monocyte chemotactic protein (MCP)-1 and macrophage inflammatory protein (MIP)-2 in peritoneal lavage fluid among the control group received a sham operation (Sham) and experimental groups 1 and $3 d$ after surgery

(Mean values and standard deviations for ten rats in each group)

\begin{tabular}{|c|c|c|c|c|c|c|}
\hline & \multicolumn{2}{|c|}{ CINC-1 (pg/ml) } & \multicolumn{2}{|c|}{ MCP-1 (pg/ml) } & \multicolumn{2}{|c|}{ MIP-2 (pg/ml) } \\
\hline & Mean & SD & Mean & SD & Mean & SD \\
\hline \multicolumn{7}{|l|}{ Post-1 } \\
\hline Sham & $56 \cdot 5$ & $68 \cdot 3$ & $43 \cdot 3$ & $16 \cdot 9$ & 61.6 & $79 \cdot 2$ \\
\hline Conv & $109 \cdot 2$ & $57 \cdot 1$ & $63 \cdot 1$ & $21 \cdot 3$ & $23 \cdot 3$ & $6 \cdot 7$ \\
\hline Gln & 80.7 & $25 \cdot 1$ & $123 \cdot 3^{*}$ & $43 \cdot 7$ & $48.0 \ddagger$ & $16 \cdot 0$ \\
\hline \multicolumn{7}{|l|}{ Post-3 } \\
\hline Sham & $27 \cdot 6$ & $16 \cdot 5$ & $27 \cdot 1$ & $13 \cdot 4$ & 30.8 & $16 \cdot 3$ \\
\hline Conv & $87 \cdot 5^{\star}$ & 57.8 & $47 \cdot 7$ & $20 \cdot 1$ & $72 \cdot 9^{*} \dagger$ & $24 \cdot 4$ \\
\hline Gln & $45 \cdot 5$ & $15 \cdot 8$ & $29.2 \dagger$ & $9 \cdot 1$ & $30 \cdot 9$ & 21.9 \\
\hline
\end{tabular}

Post-1, experimental group $1 \mathrm{~d}$ after surgery; Conv, conventional; Gln, glutamine; Post-3, experimental group $3 d$ after surgery.

* Mean values were significantly different from the other two groups on the same post-operative day $(P<0.01)$

† Mean values were significantly different from the same group on post-operative day $1(P=0.006)$.

$\ddagger$ Mean values were significantly different from the Conv group on post-operative day $1(P=0.011)$.

\section{Discussion}

The present study investigated the effect of parenteral Gln supplementation on leucocyte integrin expressions and immune cell recruitment after major surgery. Also, a gastrectomy-induced change in inflammatory mediators was evaluated. We included a Sham group in the present study. Because the Sham group also underwent jugular cannulation and laparotomy, the only difference between the Sham and Conv groups was the gastrectomy. Therefore, the difference in immune reaction in response to gastrectomy can be evaluated. We found that a gastrectomy induced early PMN CD11b/CD18 expressions on day 1, and more neutrophils were recruited to the site of injury $3 \mathrm{~d}$ after surgery. This result indicated that although rats in the Sham group also underwent operation, a higher immunological response to injury was observed when major surgery as total gastrectomy was performed in the present study.

Adhesion molecule expressions are upregulated by proinflammatory cytokines and $\mathrm{NF}-\mathrm{\kappa B}^{(22)}$. CD11a/CD18 are exclusively expressed on leucocytes, while CD11b/CD18 are abundant in $\mathrm{PMN}^{(23)}$. Excessive expression of these integrins induces an inflammatory response and tissue injury ${ }^{(23,24)}$. We did not observe difference in CD11a/CD18 among the groups; however, we found that compared with the Conv group, parenteral Gln infusion blunted the increase in PMN CD11b/CD18 on day 1 and restored it to the Sham levels on day 3 after surgery. CD11b/CD18 is reported to be a good marker of PMN activation in the inflammatory mechanism ${ }^{(20)}$. The study by Brunialti et al. ${ }^{(25)}$ showed that the expressions of CD11b/CD18 were significantly increased in patients with septic shock. Previous literature has revealed that Gln can inhibit inflammatory cytokine production and reduce nuclear binding/activation of NF- $\mathrm{B}^{(26)}$, and may, consequently, downregulate the expression of adhesion molecules in a surgical condition. The present result is consistent with a previous report which showed that Gln supplementation decreased neutrophil CD11b/CD18 expressions and plasma intracellular adhesion molecule-1 levels in a septic condition ${ }^{(10)}$.

Cytokines are peptides produced by the cells of the immune system that act as mediators of the immune response. Cytokine profiles are related to the severity of different types of infection. The cytokine profiles are determined by two functional subsets of T-lymphocytes: Th1 and Th2. Th1 cytokines, including IL-2 and IFN- $\gamma$, enhance cell-mediated immunity, whereas Th2 cytokines, including IL-4 and IL-10, enhance humoral immunity. The effects of Th1 and Th2 lymphocytes are counter-regulatory ${ }^{(27)}$. Studies have shown that overexpression of the Th2 cytokine, IL-4, is responsible for the immunosuppression associated with surgery ${ }^{(17)}$. According to our previous observations, plasma IL-2, IL-4 and IFN- $\gamma$ are not detectable in sepsis ${ }^{(28)}$; therefore, we directly measured lymphocyte IFN- $\gamma$ and IL-4 production to investigate the effects of Gln administration on Th1- and Th2-type responses after an operation. Yaqoob \& Calder ${ }^{(29)}$ found that Th1 cytokine production depends on the concentration of Gln present in the culture medium. A previous study performed by our laboratory showed that Gln supplementation enhanced IFN- $\gamma$, suppressed IL-4 production and reversed the predominant Th2 response to a more balanced $\mathrm{Th} 1 / \mathrm{Th} 2$ response during sepsis ${ }^{(18)}$. In the present study, we observed that 
compared with the Conv group, both Th1 (IFN- $\gamma$ ) and Th2 cytokines (IL-4) in the Gln group were suppressed and the extent of IL-4 decrement was greater than that of IFN- $\gamma$. Since overexpression of IL-4 is associated with operationinduced immunosuppression ${ }^{(17)}$, determining whether a lower IL-4 distribution observed after Gln feeding has a favourable effect with gastrectomy requires further investigation.

CINC-1, MCP-1 and MIP-2 are the chemokines that exhibit activities associated with inflammatory, immune and tissue repair processes. Chemokines play important roles in mediating inflammatory cell recruitment in tissues in response to infection and injury. Standiford et al. ${ }^{(30)}$, using a murine model of acute bacterial pneumonia, found that depletion of MIP-2 is associated with higher early mortality ${ }^{(30)}$. A study performed by Matsukawa et al. ${ }^{(15)}$ found that endogenous MCP-1 protected mice in a model of septic peritonitis. However, a previous study showed that serum MCP-1 levels are raised in septic patients and are even higher in those with severe sepsis ${ }^{(31)}$. Also, MCP-1 and CINC-1 were significantly increased in a severe inflammatory reaction as found in thermal injury and caecal ligation and perforation in rats ${ }^{(32,33)}$. The discrepancies among the studies may have resulted from the time required to take the measurement and the duration and location of these mediators exist in biological fluids. In the present study, we found that compared with the Conv group, the Gln group had higher MCP-1 and MIP-2 levels as soon as day 1 after surgery, indicating that Gln supplementation caused larger neutrophil and monocyte recruitment at the early stage of surgery. The chemokines were restored to the levels comparable with the Sham group at a later phase of surgery, whereas it was not with the Conv group. Gln provides a vital fuel source for the rapidly dividing leucocytes and macrophages ${ }^{(34)}$. It is possible that whenever Gln is available for use, neutrophils and macrophages are activated rapidly in response to the injury as observed on post-operative day 1 and returned to the basal levels afterwards.

In conclusion, the present study showed that administration of TPN-containing Gln affected several aspects of the immune response that differed from those seen with Conv TPN. We found that, compared with the Conv group, Gln supplementation attenuated PMN integrin expression as soon as day 1 and limited lymphocyte cytokine release on day 3. In addition, Gln-enriched parenteral nutrition caused larger neutrophil and monocyte recruitment at the early stage, and this was restored to Sham levels at a later phase of surgery, whereas it was not with the Conv parenteral nutrition. This is likely that Glnenriched parenteral nutrition induced an earlier more intensive and rapid immune response to injury than the Conv parenteral nutrition.

\section{Acknowledgements}

The funding of the present study is totally supported by research grant NSC95-2314-B-002-157 from the National Science Council, Republic of China. No financial support from any other sources. The authors are not the employees or consultants associated with any commercial companies and there is no conflict of interest in the present study. M.-T. L. contributed to the concept of the study and prepared the manuscript; S.-Y. C., S.-S. T., M.-Y. W. and M.-H. W. did the data analysis and interpreted the data; and S.-L. Y. designed the study, prepared and revised the manuscript. All authors have read and approved the final submitted manuscript.

\section{References}

1. Delany HM, Demetriou AA, Teh E, et al. (1990) Effect of early postoperative nutritional support on skin wound and colon anastomosis healing. J Parenter Enter Nutr 14, 357-361.

2. Bragelmann R, Armbrecht U, Rosemeyer D, et al. (1996) Nutrient malassimilation following total gastrectomy. Scand J Gastroenterol 218, 26-33.

3. Braga M, Gianotti L, Andrea V, et al. (1998) Artificial nutrition after major abdominal surgery: impact of route of administration and composition of the diet. Crit Care Med 26, 24-30.

4. Parry-Billings M, Evans J, Calder PC, et al. (1990) Does glutamine contribute to immunosuppression after major burns? Lancet 336, 523-525.

5. Wilmore DW (2001) The effect of glutamine supplementation in patients following elective surgery and accidental injury. J Nutr 131, S2543-S2549.

6. Kudsk KA, Wu Y, Fukatsu K, et al. (2000) Glutamine-enriched total parenteral nutrition maintains intestinal interleukin-4 and mucosal immunoglobin A levels. J Parenter Enter Nutr 24, 270-275.

7. Wischmeyer PE, Kahana M, Wolfson R, et al. (2001) Glutamine reduces cytokine release, organ damage, and mortality in a rat model of endotoxemia. Shock 16, 398-402.

8. Gianotti L, Alexander JW, Gennari R, et al. (1995) Oral glutamine decreases bacterial translocation and improve survival in experimental gut-origin sepsis. J Parenter Enter Nutr 19, 69-74.

9. Yeh SL, Lai YN, Shang HF, et al. (2004) Effects of glutamine on innate immune response in rats with gut-derived sepsis. $\mathrm{Br} \mathrm{J}$ Nutr 91, 423-429.

10. Yeh CL, Hsu CS, Yeh SL, et al. (2006) Dietary glutamine supplementation reduces cellular adhesion molecule expression and tissue myeloperoxidase activity in mice with gut-derived sepsis. Nutrition 22, 408-413.

11. Novak F, Heyland DK, Avenell A, et al. (2002) Glutamine supplementation in serious illness: a systemic review of the evidence. Crit Care Med 30, 2022-2029.

12. Driscoll KE, Carter JM, Hassenbein DG, et al. (1997) Cytokines and particle-induced inflammatory cell recruitment. Environ Health Perspect Suppl 5, 105, 1159-1164

13. Takano K \& Nakagawa H (2001) Contribution of cytokineinduced neutrophil chemoattractants CINC-2 and CINC-3 to neutrophil recruitment in lipopolysaccharide-induced inflammation in rats. Inflamm Res 50, 503-508.

14. Greenberger MJ, Strieter RM, Kunkel SL, et al. (1996) Neutralization of macrophage inflammatory protein-2 attenuates neutrophil recruitment and bacterial clearance in murine Klebsiella pneumonia. J Infect Dis 173, 159-165.

15. Matsukawa A, Hogaboam CM, Lukacs NW, et al. (1999) Endogenous monocyte chemoattractant protein-1 protects mice in a model of acute septic peritonitis: cross-talk between MCP-1 and leukotriene $\mathrm{B}_{4}$. J Immunol 163, 6148-6154.

16. Lennard TWJ, Shenton BK, Borzotta A, et al. (1985) The influence of surgical operations on components of the human immune system. Br J Surg 72, 771-776.

17. Ayala A, Deol ZK, Lehman DL, et al. (1994) Polymicrobial sepsis but not low-dose endotoxin infusion causes decreased splenocyte IL-2/IFN-gamma release while increasing IL-4/IL10 production. J Surg Res 56, 579-585.

18. Yeh CL, Hsu CS, Yeh SL, et al. (2005) Dietary glutamine supplementation modulates Th1/Th2 cytokine and interleukin-6 expressions in septic mice. Cytokine 31, 329-334. 
19. Springer TA (1990) Adhesion receptors of the immune system. Nature 346, 425-434.

20. Smith S, Joneckis C \& Parise L (1993) Regulation of vascular integrins. Blood 81, 2827-2843.

21. Ohta A, Motohashi Y, Sakai K, et al. (1998) Dietary fructooligosaccharides increase calcium absorption and levels of mucosal calbindin-D9k in the large intestine of gastrectomized rats. Scand J Gastroenterol 33, 1062-1068.

22. Jersmann HP, Hii CS, Ferrante JV, et al. (2001) Bacterial lipopolysaccharide and tumor necrosis factor alpha synergistically increase expression of human endothelial adhesion molecules through activation of NF-kappaB and p38 mitogen-activated protein kinase signaling pathways. Infect Immun 69, 1273-1279.

23. Henderson RB, Lim LHK, Tessier PA, et al. (2001) The use of lymphocyte function-associated antigen (LFA)-1-deficient mice to determine the role of LFA-1, Mac- 1 and $\alpha 4$ integrin in the inflammatory response of neutrophils. J Exp Med 194, 219-226.

24. Ulbrich H, Eriksson EE \& Lindbom L (2003) Leukocyte and endothelial cell adhesion molecules as targets for therapeutic interventions in inflammatory disease. Trends Pharmacol Sci 24, 640-647.

25. Brunialti MKC, Martins PS, de Carvalho HB, et al. (2006) TLR2, TLR4, CD14, CD11b and CD11c expressions on monocytes surface and cytokine production in patients with sepsis, severe sepsis, and septic shock. Shock 25, 351-357.

26. Singleton KD, Beckey VE \& Wischmeyer PE (2005) Glutamine prevents activation of NF-kappaB and stress kinase pathways, attenuates inflammatory cytokine release, and prevents acute respiratory distress syndrome (ARDS) following sepsis. Shock 24, 583-589.

27. DiPiro JT (1997) Cytokine networks with infection: mycobacterial infections, leishmaniasis, human immunodeficiency virus infection, and sepsis. Pharmacotherapy 17, 205-223.

28. Yeh SL, Yeh CL, Lin MT, et al. (2001) Effects of glutaminesupplemented total parenteral nutrition on cytokine production and $\mathrm{T}$ cell population in septic rats. J Parenter Enter Nutr 25, 269-274.

29. Yaqoob P \& Calder PC (1997) Glutamine requirement of proliferating T lymphocytes. Nutrition 13, 646-651.

30. Standiford TJ, Kunkel SL, Greenberger MJ, et al. (1996) Expression and regulation of chemokines in bacterial pneumonia. J Leukoc Biol 59, 24-28.

31. Bossink AW, Paemen L, Jansen PM, et al. (1995) Plasma levels of the chemokines monocyte chemotactic proteins-1 and -2 are elevated in human sepsis. Blood 86, 3841-3847.

32. Gauglitz GG, Song J, Herndon DN, et al. (2008) Characterization of the inflammatory response during acute and postacute phase after severe burn. Shock 30, 503-507.

33. Miyakawa H, Kira S, Okuda K, et al. (2008) Olprinone decreases elevated concentrations of cytokine-induced neutrophil chemoattractant-1 in septic rats. J Anesth 22, 27-31.

34. Newsholme EA, Crabtree B \& Ardawi MS (1985) Glutamine metabolism in lymphocytes: its biochemical, physiological and clinical importance. Q J Exp Physiol 70, 473-489. 